\title{
On the Explicit Function of Life within a Physical Universe
}

\author{
Annette Grathoff 1,2,3 \\ 1 GSIS, 1220 Vienna, Austria; annette.grathoff@gsis.at \\ Design Institute, 80797 Munich, Germany \\ BCSSS, 1040 Vienna, Austria
}

check for updates

Citation: Grathoff, A. On the Explicit Function of Life within a Physical Universe. Philosophies 2021, 6, 59. https://doi.org/10.3390/ philosophies6030059

Academic Editors: Rainer

E. Zimmermann and Marcin J. Schroeder

Received: 1 May 2021

Accepted: 13 July 2021

Published: 17 July 2021

Publisher's Note: MDPI stays neutral with regard to jurisdictional claims in published maps and institutional affiliations.

Copyright: (C) 2021 by the author. Licensee MDPI, Basel, Switzerland. This article is an open access article distributed under the terms and conditions of the Creative Commons Attribution (CC BY) license (https:// creativecommons.org/licenses/by/ $4.0 /)$.

\begin{abstract}
To describe the meaning of functionality in a universe before life evolved, existing etiological and systemic accounts of function are evaluated. Since the theory of function is only applicable in context with living beings and artifacts used by living beings and therefore cannot predict how a prebiotic form of functionality could evolve, a maintenance account for functionality is proposed. This account ascribes functionality to a structurally disposed property that increases the probability of maintenance or recurrence of the property in the surrounding selective environment. With the help of the maintenance account and a concept of physical information comprising kinetic and structural types of information, possible evolutionary processes preceding the evolution of life are explored. As important mechanisms in abiotic and prebiotic evolution, linear and non-linear mixing processes, as well as dynamics of solitary waves, are identified. Before the question of the meaning of life in prebiotic environments is renewed and an educated guess based on the elaborated arguments is made on the progress of evolution under the influencing impression of the living state, the evolution of functionality in different selective contexts is analyzed.
\end{abstract}

Keywords: theory of function; physical information; non-linearity; wave dynamics; soliton; prebiotic evolution; kinetic information; structural information

\section{Introduction}

The ongoing discourse in the philosophy of science over more than half a century corroborated the fact that the human idea of function and functionality is deeply grounded in a view of function and functionality of tools on the one hand. On the other hand, it became clear that the wide applicability of the terms makes them very useful for analysis of effects in categories that range from patterns (e.g., of behavior) to species (in biological function), types of tools (artifact function) and farther. Function stands for what members of a category all "are supposed to" do, that is, "are supposed to" be capable of, and intuitively the performance of a function is linked to the presence of specific processes and structures. The normatively grounded ascription of function is usually tightly coupled to a teleological dimension by trend. To avoid mistaking the inference to an explanation for an explanation itself, the philosophy of the sciences community developed emphases for scientifically useful accounts on function [1-10]. In these, both the descriptive as well as the normative and teleological dimensions of the intuitive concept are elucidated. The explanations for the intuition "are supposed to" were developed in three main theories of function (called accounts): The framework comprises causal history aspects that ground the current presence of a function bearer in its function in past selective processes (etiological accounts) $[7,10]$. It comprises aspects of causal contribution to a systemic capacity that ground the current presence of a function bearer in its function inside a system of higher organization which it, at the same time, belongs to and helps to maintain (organizational accounts) $[1,4,5,8]$. It also comprises aspects of enhancing biological fitness that ground the current presence of a function bearer in its systemic contribution to improving life chances according to the theory of natural selection (etiological/systemic selected-effect accounts) [2,3]. Aspects from all three theoretical approaches taken together are capable of 
explaining almost any functionality intuitively attributed to a given structure. To further emphasize the context dependence of function and thereby the complementarity of a "user of function" and a "provider of function", it is useful to additionally consider von Uexküll's concept of environment ${ }^{1}$ [11-13] and the concept of affordance [14-16]. Although this notion of affordance is implicit in etiological, selected-effect and also organizational accounts, it is worth being pointed out separately. In cases where a structurally disposed property [4] is-retrospectively - desired but is not what the function bearer was originally "supposed to do", or, is not the function for which an artifact was intended for by the designer, its value becomes obvious: BROWN and BLESSING [15] pointed out that this view is " $(. .$.$) allowing affordances to be recognized from experience, to be learned, and to be$ inferred by analogy."

The etiological account, organizational account, selected effects account and emphasis on the complementarity aspect contribute to explaining why people tend to observe functionality in structures; why functionality tends to be connected to certain structures by means of structurally available ways of having an effect. The fact of being functional or of having a function in a certain context is thus connected to the maintenance of the function bearer by its surrounding environment ${ }^{2}$. The function bearer is a structural disposition, and it can either be a very complex one which is itself an individual environment with selective capacity or a not very complex piece or part directly subordinated to the selective environment it is enclosed into. In their refined organizational account, Mossio, SABORIDO and MORENO [5] described function in complex biological systems: living beings as " $(. .$.$) organizationally closed and differentiated self-maintaining systems, possess$ the necessary properties for adequately grounding both the teleological and normative dimensions of functions in their current organization." Organisms are complex individuals that evolved multiple functions and have an environmental character for a lot of functional processes inside their bodies. Their bodies as their inner environments are selective on metabolic biochemical and electromagnetic dispositions and produce functions that are under the selective pressure of the surrounding environments of the organism. Artifacts and environmental niches to use are selectively chosen by living individuals, and if they are considered functional, they might be maintained in "use". Likewise, aspects of their structure might even be reproduced in (transferred to) similar contexts. The main cause for maintenance inside the surrounding environment is what has been discussed in the three accounts: Maintenance because of preserved entries of past proves of value inside the selector, maintenance because of contribution to a maintaining system as its active part and maintenance by the laws of natural selection. Functionality according to the maintenance account—proposed here as a useful addition to function theories-means a structurally disposed property, a structural disposition, which is leading to maintenance of this property in a selective environment. The physical basis for this account is elaborated in this work. There are strengths but also weaknesses of every account, and thus the most promising approach to the notion of function in sciences is to keep them all in mind: The etiological-historical account, e.g., suffers from its inherent incapacity of analyzing current outputs of structures that are functional according to the historical record but lost function and likewise of novel structures that would intuitively be called functional or better functioning but for which yet no record exists to explain the intuition [7]. The organizational account taken for itself due to its focus on present activity suffers from the difficulty to differentiate elements of a system that are contributing actively from those that accidentally got into the system and are just neutrally (or even detrimentally) acting inside. On the other hand, the organizational account seems well equipped to treat living individuals as functional organs of a supraindividual organism. The systemic/etiological selected-effects account suffers from its focusing on just one type of selective regime namely the one described by Darwin as a source of natural selection excluding its application in explaining the function of designed cultural artifacts [9]. The maintenance account taken for itself does not have the capacity of differentiating between different causes that lead to maintenance of a structurally disposed property or a system of nested environments 
(such as a body of a living being) inside one and the same selection regime. Viewed from an evolutionary perspective, the maintenance of a structure that is detrimental for the selecting individual or environment but that cannot be recognized by its sensory capacity or (spatio-temporal) resolution, being a function of that structure, is logically correct; capacity to hide and to disguise or to live unrecognized by a hosting organism is subjected to adaptive and co-evolutionary processes, and parasites not only have evolved functional adaptations ${ }^{3}$ but also drive evolution. In the human socio-cultural environment where decision making is additionally influenced by moral criteria of the economic system and a communication system with a huge long-term storing capacity of observations to name just a few, further differentiation becomes necessary. Considered together, the respective weaknesses of a single theory are each complemented by the strengths of (one of) the others. Existing accounts, nevertheless, occasionally prove their uncertainty of specifying a function bearer in view of that selective regime according to the selection parameters of which it is maintained or even repeatedly produced ${ }^{4}$. Often the selection criteria are not considered or are not important because exact mechanisms are multilayered and complex and it is the result that counts.

All accounts except the maintenance account are closely connected to the existence of life. The aspect of usefulness as a tool and affordance which is known from cultural function analysis in medical or technical contexts often plays a role in the definition of functionality-more or less hidden. This is totally understandable, as long as one is dealing with complex systems with goal-directedness which functional elements are serving. It is deeply grounded in human intentional thinking and decision making. If, on the other hand, the origin of directedness of evolution and development in systems is examined, if the question is which of the structural dispositions that are maintained are causing the directedness, then the maintenance account is most useful. Selective regimes considered in the accounts reach from the system of natural selection for biological species, over internal selection inside living individuals' bodies involving repair mechanisms and the immune system, which are nested in surrounding environments due to their contribution to the fitness of the hosting bodies, to intentional and culturally based selective systems of individuals and cultures, evaluating, among others, according to usefulness and pleasantness.

From the maintenance-focused view, function defined in a negative way is the property that does not lead to dissolution of its structural disposition or to being extinguished inside an environment. When this property is observed, it usually is not analyzed any further. Function can then serve as a term for a property or invariant process that is being maintained inside a given environment. The maintenance by the environment can be quantified by thresholds if the selective parameter that is responding to the property is known. In the case of approaching the threshold value, the probability of maintenance decreases for the property while the probability of choosing to maintain other carriers of the same or better fitting property instead increases. The property becomes a role that can be played by several similar structures. One of the goals of this work is to strengthen the already strong basis of theories of function, so that one is able to consider functionality as a property that evolved alongside life but the basic forms of which already exist in prebiotic physico-chemical contexts and so that the question of what could be the function of life as one (special) state in a world of different states of matter and fields can be approached. For physical systems, information viewed as "a difference that makes a difference" interpreted along the lines of Bateson's famous quote $[17,18]$ leads to two important and mutually corresponding questions:

- How does a difference arise in physical structures?

- How can a difference be made in physical structures (triggering a difference in its configuration that subsequently leads to new ways of interacting with its environment)?

- These questions are elaborated and discussed in the following so that the inherent question in the title about the meaning of functionality in different environments can be addressed in the discussion. 


\section{Results}

\subsection{How Can a Developed Physical Difference Make a Difference by Functionality?}

When thinking about the proposed question, most probably the first thought is about particles and particulate structures and how they can change their form and disposed properties by plastic deformation and chemical reactions. What kind of influence can structurally disposed properties in a particle have on the course of evolution of the particle's maintenance in its environment? Can those properties be self-referential to their own state? There exist already good models for how a particulate structure can self-organize relying on some kind of coding and modularity-as is the case in nucleic acids, amino acids and living organisms. To explore the explanatory power of the maintenance account, the notion of function is used as a tool to discover self-referentiality in objects that at first are not believed to show such. We are going to consider the wave dynamic formation of transiently stable and mildly predictable parcels of force and information inside energy density fields, no matter whether the carrying medium is the electromagnetic vacuum or matter, in any state of condensation (gaseous, liquid, solid and intermediaries). Non-dispersive wave packets or localized pulses are special for maintaining their identities even when they undergo collisions with each other. This particle-like behavior gave them the name soliton. However, solitons are also special for showing that particle-like behavior of packets of wave-frequency components demands a compensation of natural wave dispersion ${ }^{5}$ behavior, which - in contrast to stability of atoms and elementary particles-appears to be a property of the medium in which the wave is excited at least as much as it is a property of the wave itself. A soliton cannot be viewed separately from its medium.

Solitons became known by an observation of RUSSELL (1808-1882) in water (Union Canal system). One might already know of the narrative of him observing the generation of a wave by a canal boat pulled by horses stopping suddenly, realizing the special character of the wave and then following its course over more than a mile on horseback. After this observation, Russell built his own "soliton generator" to further understand and analyze the interesting phenomenon (see for example [20]). The canal was in fact-and shallow canals tend to be in general-one of the structural media that support solitons: from observations in open waters such as ponds where one throws in a pebble to generate cyclic wave fronts spreading out (For the sake of completeness: in addition to dispersion, the inverse square law is important for this effect), one would expect excited waves to quickly lose their form, dispersing into more and more disconnected diminishing wiggles on the surface due to different frequency components having different velocities. Then the character of an "object" with a form or an entity of coupled components normally is lost. When non-linear media such as the shallow body of water in Russell's canal enable excitation of localized pulses, or "wave packets", they somehow counteract dispersion respectively diffraction, eliminating phase differences between the components. How can a medium eliminate mutual phase differences in wave packets? KORTEWEG and DE VRIES realized that the phenomenon described by Russell required an unusually large amplitude and that the medium's non-linearity must be grounded in a different behavior to waves of different amplitudes [21]. The ship's speed is the decisive factor because for speeds of water dragged along by the ship which are much lower than a critical threshold speed, i.e., slower than waves radiated from the ship, those linear waves will effectively carry away energy, not allowing the steepening to higher amplitudes [22]. However, if the ship's propagation speed $^{6}$ reaches the critical threshold, a local radiation wave can grow as the energy acquired from a local fluid at the rate of work by its motion keeps accumulating. Local waves with a small initial amplitude thus are fueled by energy from the disturbance over a relatively long time. If a local wave reaches a certain threshold magnitude, the increase in phase speed with increasing amplitude-enabled by the non-linear behavior of the medium to different amplitudes-is then sufficient to make the wave break away from the disturbance, thus freeing it as a new solitary wave propagating forward with a phase velocity dependent on the amplitude it gained. What is particularly interesting in the case of the excitation of such a discrete "precursor soliton" entity is that it has been generated 
by a steadily moving disturbance in the medium (i.e., the moving ship or the accelerated flow of water propagating after the ship had stopped). It even has been observed that many solitons may be generated subsequently ([23] and references therein). What the nonlinearity of the medium enables in the case of Russell's soliton is therefore the following. Out of a forcing steady flow, a continuous and periodic sequence of solitary waves or solitons can be generated, i.e., some kind of differential enhancement or perpetuation effect on the excitation [23]. Due to its topological structure, the canal enhanced the amplitude of some frequencies and dampened the amplitude of others, leading to a distribution of energy in a non-averaged, frequency-selective manner. The mutual phase differences in components of the wave packet that would in the linear case have led to an averaging out of distinct rises in the amplitude of certain frequencies are eliminated in an indirect way: features of its geometry forbid certain frequencies ${ }^{7}$ If the topological structure was different in critical places, the same excitation event would allow the frequencies that are forbidden in the canal to develop sufficient amplitude to be measurable. Transformative capacity of structures on intensity distribution and the temporal pacing of gradients and their energy flows into periodic oscillatory motion enabled by their special geometry and topology plays a very important role in thermo-acoustic self-oscillators [24,25] or in musical self-oscillators, such as reed instruments and flutes [24,26]. In a thermo-acoustic oscillator, the acoustic oscillation of gas is encouraged if heat is injected when the gas is warmed by adiabatic compression, and/or heat is removed when the gas is cooled by adiabatic expansion. Similarly, in a reed instrument such as the clarinet, the reed by its non-linearity lets the high pressure from the breath of the player join the high-pressure part of the sound wave but not the low-pressure part, increasing the strength of the resonance in the cavity and generating a tone at the resonant frequency. In both cases of self-oscillation, the elasticity of a "spring" component in the structure, i.e., of a differentially accelerating component, is varied in such a way that the overall oscillation is fueled by a continuous flow. A differential acceleration or differential enhancement as it is given by the non-linearities of media or artifact structures discussed is a physical basis for selection and selectivity on dynamics. Selecting out different components of wave packets in its effect is not necessarily what selecting different particles from a system would be. When talking about selection, the first intuition is to take away or choose out of... In wave packets or frequency spectra of traveling waves, a selective enhancement or dampening of a frequency has no influence on its potential presence. It does not irreversibly take away but only influences the distribution and local observability of the frequency. Intuition has to first get used to the different selection mechanism and effects in wave dynamics from experience. It is not accustomed to mechanics where disturbing an averaging process by stopping some destructive interference leads to gaining new possibilities here at the cost of elsewhere stopping constructive interference, thus losing possibilities inside a possibility spectrum. The main selective mechanism in wave dynamics is interfering with interference processes. Interference in wave dynamics is a disturbed averaging process of amplitude height given by coherence between waves. It is a linear process; the spectrum is not altered, but the intensities of possibilities (frequencies) are.

In non-linear media, most of the excited waves are changed in their spectra respectively bandwidths. Contrarily, linear mixing in linear media is the superposition of things treated as individuals, which still allows for recognition of each of the individuals and which keeps any individual separable and unchanged. A linear mixing is for example the additive mixing of colors. If the "things" perform cyclically repeating, periodic motions for which a point in the cycle relative to another point in the same cycle can be defined as a phase, such as in waves, the relationship between the cycles becomes important. This relationship, the relative phase between units, can be incoherent, i.e., over a sufficiently large number of units, the regions of local constructive and destructive interference fluctuate so fast that an average value for intensity emerges. The relative phase can also be coherent, enabling a stable pattern of interference regions. 
In coherent relative phase, constraining of wave dynamics with structures, the dimensions of which are at the scale of the waves' wavelengths in the structures, lead to distinct patterns of enhanced and dampened intensity, i.e., fringe patterns (diffraction patterns). Structural objects with this property are usually referred to as diffraction gratings. Interference is also important in the generation of standing waves and cavity resonance. Interfering with interference processes is possible by coupling and decoupling phases, altering the relative phase or by manipulating points of interference nodes and antinodes.

One common differential enhancement mechanism is the resonance amplification of certain frequencies respectively harmonic components of a fundamental frequency in resonant cavities. Influencing resonance is thus possible by altering the node and antinode distribution for waves and altering the fundamental, e.g., by re-scaling the cavity. Another is the manipulation of the decay time of resonances by adding leakage at points where certain excitable frequencies have their antinodes, strengthening amplitudes of others with a node at that spot. Thereby harmonics of a fundamental can also be selectively dampened, without altering the fundamental itself. Both processes are clearly described in [26].

In the sense of evolutionary biologist MAYR who developed DARWIN'S "descent with modification" concept of selection [27] further, embracing the (relatively new knowledge on) genetic information, selection in biology, i.e., natural selection can be defined as "(...) differential perpetuation of genotypes" [28]. Taking this accepted definition for biological selection into account, differential acceleration or differential enhancement by interfering with interference in physical systems - similar to differential permeability of physical sieves (although working by very different mechanisms as stated above)—can be construed as physical selection. This ascribes a basal selective capacity to processes interfering with interference dynamics in physical systems. Linear processes do not dissipate energy in exchange with surroundings. Absorption and emission events happen only internally and have no influence on the average of ensembles. Non-linear processes are characterized by dissipation and irreversible transformations. Topological features in the medium are altered; they are altered in a spatial pattern that is proportional to the spatio-temporal pattern of intensity (or radiance ${ }^{8}$ ) transported in the wave field. The changes in the medium take place due to (e.g., photochemical) reactions fueled by the spatio-temporally irradiated (photon) energy as power/area- (intensity $\left[\mathrm{J} / \mathrm{sm}^{2}=\mathrm{W} / \mathrm{m}^{2}\right]$ ) or intensity related available energy. Subsequently, properties important for interactions with fields such as the one that caused the alterations are altered. Those properties comprise absorption, transmission, reflection and the complex medium property refractive index (RI). The RI is mathematically defined as the relation between traveling speed of the phase (in the case of the electromagnetic field being the wave field under consideration: the speed of light in vacuum, c) and the traveling speed inside the medium, $\mathrm{v}$. RI c/v, and in most known naturally occurring media, it is smaller than 1 . The micro-dynamics behind the effect of the RI of a medium on an incoming wave are quantum mechanical by nature and are not delved deeper into here, but a simple macroscopically observable detail is helpful for visualization: Remember that we discussed interference with interference being the main mechanism of altering the intensity distribution structure of a wave field. In classical optics, what is important in a wave field for the average intensity (incoherent relative phase) or the diffraction pattern (coherent relative phase) spatio-temporally "coded" intensity distribution transmitted to a screen is the front hitting the screen at a distance to the field's source, called the wave front. When a wave front hits a screen, depending on the complex RI of the screen material medium, part of the wave front will be reflected, part of it will be transmitted and other parts of it might be absorbed. In reflection, as in other cases, classical optics uses the model of the so-called Huygens's elementary wave mechanism. The details can be found in any physics textbook comprising classical optics and are worth deeper study, but for the discussion here, the important message from the model is that it is possible to construe any part of a wave front as a source for an elementary, i.e., spherical wave (in three dimensions). This is valid for the original wave front as well as for its reflection after it hit—and possibly interacted with—the medium. This is the crucial point. 
If the medium in interaction altered the phase of a reflected wave, the intensity at that point of the medium as a source for the reflected wave front might be altered due to interfering with interference, compared to the wave front that came in. In the case of light, when the light goes from a low-RI medium to a high-RI medium (air to glass), the reflection undergoes a 180-degree phase change. When the light goes from a high-RI medium to a low-RI medium (such as glass to air), it does not undergo a phase change. This is analogous to the case of a vibrating string (from footnote 7 ) as an example for the generation of a standing wave: At an edge of the medium that is held fixed (i.e., the point is not allowed to displace at amplitude as every other point in the medium), the reflected wave flips over (corresponding to a 180-degree phase change). At an edge of the medium that is free to displace, the reflected wave does not flip over [30]. This becomes relevant at any point in the medium where there is a sufficiently large region of altered RI while the wave is propagating through. Especially interesting is the case of the incoming wave interfering with its reflected "copy" since this is an example for a coherent relative phase, and the interference defines the image of the reflector, i.e., the incoming wave as a reference wave plus the modulation on the phase done during reflection. In a first summary, it can be said that:

- Linear interference influences the intensity distribution between and local observability of frequencies of a given spectrum.

- Depending on the relative phase, the intensity of frequencies inside the given spectrum can at a screen at a certain distance to the source of the wave front attain an average value or an image of the primary source modulated by a reflector ${ }^{9}$ (and possibly a noisy transmission medium, e.g., air) at that frequency as a diffraction pattern.

- When a wave front transmitting a diffraction pattern as a spatio-temporally "coded" intensity distribution in the field hits a target surface which is sensitive for some of its frequencies, energy is not simply transmitted but is transmitted with information.

A medium that has been altered by transmission of informed energy can non-linearly change and thereby be altered in properties decisive for subsequent interactions with fields. One possible mechanism for this is that the oscillation of dipoles in molecules induced by a wave affects the momentary local charge distribution (polarization) in the medium and thereby causes local changes in the refractive index.

Thereby non-linear processes plus linear interference can become the means by which an image, which is itself a "non-physical" object, can display a primordial type of selfreferentiality. The importance of this finding is further elaborated in this paper, using the maintenance account to functionality. In principle, alterations of the fundamental and the composition of the spectrum are also possible, leading to altered properties as a re-emitting source. This is, for example, possible when the triggered dissipative reaction alters geometries of molecules or the scale of cavities in the receiving material. In this case, the self-referentiality is not given as it is for the process of transmitting an image, but resonances can selectively enhance particular similar frequencies.

Before further elaborating the possible influence of wave-carried images as a primordial physical self-referentiality on the course of evolution and before deepening the discussion of functionality evolving and the importance of the maintenance account, the meaning and importance of non-linearity in general for selective processes transmitted by wave dynamics are further explored.

\subsection{Making a Difference by Non-Linear Wave Guiding and Mixing between Frequencies}

When modeling the relation between dispersive energy loss (dampening) and nonlinearity of amplitude distribution between available modes, such as the steepening to higher amplitude, in a non-linear partial differential equation (e.g., the equation of KORTEWEG and DE VRIES (1895)), the stabile trade-off that is observable as a soliton is an abstract mathematical solution of the equation. Unfortunately, the lack of human intuition regarding the mathematical link to the physical system is a drawback, often observed when describing motion structures. One understands the soliton phenomenon by being cable 
of modeling the dynamics and by assuming that the structure associated with the repeatable observation of the phenomenon has as its function $(\mathrm{f}(\mathrm{x})$-being a simple relational function - the generation of the dynamics given the context. Fortunately-while examining more and more occurrences of the phenomenon and the respective contexts using scientific methods to finally arrive at a more intuitive level of understanding-humans can keep inspiring their creativity and those of their peers by beautifully poetic pieces of wisdom such as: "Solitons have the, seemingly, innate ability to solve non-linear system [sic], much like a travelling plane wave seems to solve all the linear ones:) [20]". The intuitive rationale given in the quote is that the structure of a soliton mathematically irrespective of its difference to that of a linear traveling wave is similar to the latter in being a stable solution of dynamic equations. Both are describable as solutions to an algorithmic equation. The description of the soliton cannot easily be followed with the Newtonian picture of a largely empty space in which single mass carriers are moving subjected to forces. This picture is what resonates best with our daily routines since we experience the airy space through which we are moving as giving way to our motion, largely independent of its state (maybe there is an exception when we try to move against the direction of the blow of very strong wind). Charges hardly matter macroscopically, and for our motion, the structure of the surface of the runway is more crucial than the state of the airy medium. In the case of the optical soliton traveling the optical fiber, the refractive index of the medium can be locally changed by the action of the electromagnetic wave traveling through, so that the medium becomes the transmitter of light action on light propagation, thus influencing the refraction of follow-up waves and the modulating light wave's own dispersion by induced change in the refractive index [21]. This is called wave guiding and is a non-linear process of frequency interaction enabled by photorefractive dielectric and polarizable media.

Non-linear wave interaction is always facilitated by a non-linear medium through which the waves travel. Compared to the initial spectrum (called the reference wave in the following) the altered medium can indeed make waves that have new frequencies and thereby new properties when interacting with matter. Solitons are a stable solution of non-linear partial differential equations, but non-linear media as "simple" facilitators of non-linear mixing of waves are essential for their evolution. In non-linear mixing, the input-output relation is asymmetrically distorted by the medium. Non-linear mixing is a superposition of things where the individual character and the separability (at least transiently) get completely lost. It is irreversible as long as no memory plus a non-adiabatic recovery process are involved. Both mean a considerable amount of action. For non-linear processes to occur, the medium where the process is happening needs to inject into or extract energy from the mixing components, re-distributing system energy.

The range of immediate physical changes through linear superposition and interference possible is limited compared to non-linear mixing processes where substantially new components, e.g., new excitable frequencies can emerge (here, the term emergence can be described mathematically). To better visualize the difference made by the influence of non-linear media compared to purely linear mixing, the example of audible beating is chosen. It is chosen because the sound waves of tuning forks often used to show the beating phenomenon can be easily modeled as simple sinusoid waves, which greatly supports the understanding of the mixing process.

The transition from the interference-borne result of linear mixing in beating to the occurrence of new frequencies due to a non-linear process is discussed. Beating occurs, e.g., when two tuning forks sound together, one being slightly out of tune. Additionally, a non-linear process occurs (where it occurs exactly has been and still is a matter of debate and is discussed below) so that from two sinusoid waves that are almost identical (called $f_{1}$ and $f_{2}$ ), new frequencies are generated and can be perceived. When the new frequency which is the difference between the two non-linearly mixed frequencies is slow enough, humans do not hear it as a tone but as a periodic oscillation in the sound intensity of the summation tone, i.e., as a beating. When above $\sim 20 \mathrm{~Hz}$, the human auditory threshold, the difference frequency is heard as a tone too, and the frequency mixing becomes observable. 
The two similar sine waves oscillating at $\mathrm{f}_{1}$ and $\mathrm{f}_{2}$ (colored blue and green, respectively, in Figure 1) are linearly superposed. Due to constructive and destructive interference in their dynamically evolving phase relation, the amplitude periodically increases and decreases and does so with a frequency (colored orange in Figure 1) that is the sum frequency of $\mathrm{f}_{1}$ and $\mathrm{f}_{2}$. As long as no non-linear medium is distorting the superposition, no signal is generated at the sum frequency. The Fourier transform of the time domain to the frequency domain only shows the two original frequencies $f_{1}$ and $f_{2}$, and thus this is no new acoustic signal yet. Likewise, the difference frequency (colored red in Figure 1) is not appearing in the Fourier transform, just the intensity oscillation in the time domain. To arrive at the generation of a sum and a difference frequency signal, a non-linear distortion needs to occur as is shown in Figure 2. The mathematical function describing the mixing in the non-linear medium, the so-called transfer function, needs to be of quadratic order or higher; that is, it must contain exponents that are at least quadratic.

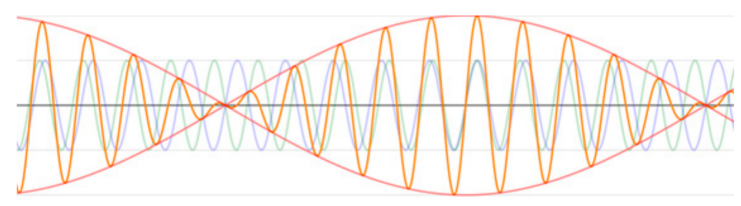

Figure 1. The superposition of two sine waves with similar frequencies $f_{1}$ and $f_{2}$ (blue and green) linearly causes an interference frequency sinusoid at the sum frequency $\left(f_{1}+f_{2}\right) / 2^{10}$ (orange) and another sinusoid which is the envelope of $\left(f_{1}+f_{2}\right) / 2$ modulated at difference frequency $\left(f_{1}-f_{2}\right) / 2$. The Fourier transform contains only the two frequencies $f_{1}$ and $f_{2}$. Picture taken from [31].

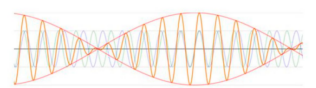

(a)

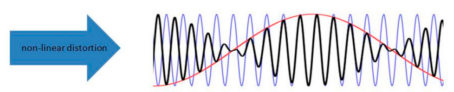

(b)

Figure 2. A non-linear distortion happening inside a non-linear medium can generate a perceivable signal of the non-linear frequencies: (a) out of the beating between $\mathrm{f}_{1}$ and $\mathrm{f}_{2}$ under the influence of a non-linear distortion in the medium $(b)$ the non-linear frequencies can emerge: The sum frequency $\left(f_{1}+f_{2}\right) / 2$ (dark blue) and the difference frequency $\left(f_{1}-f_{2}\right) / 2(r e d)$ modulating its envelope. The modulated frequency $\left(f_{1}+f_{2}\right) / 2$ is the carrier frequency of the modulating frequency. The Fourier frequency domain contains only the two non-linear frequencies $\left(f_{1}+f_{2}\right) / 2$ and $\left(f_{1}-f_{2}\right) / 2$. Pictures taken from [31,32]. Source: Mike Run.

Interestingly, the beating of the two tuning forks (given the difference frequency is above the auditory threshold of the listener) can be heard. There needs to be a nonlinear process happening somewhere facilitating the multiplication between frequencies. The air can be excluded as a source for the mixing since it is assumed to be a largely isotopic, linear medium. The location of the non-linear medium, or, the location where the mixing occurs has been a matter of centuries-long debate [33]. Hermann von Helmholtz (1821-1894), who was a talented musician and an expert in music theory, modeled the mechanism of the human ear as a Fourier analyzer. He associated the location of the frequency perception on the human basilar membrane with corresponding sinusoidal frequencies. To explain the beating, von Helmholtz associated the transfer of vibrations from the auditory ossicles to the cochlea and its basilar membrane and established the "distortion theory", an aspect of the theory of the existence of "objective combination tones". He also did a lot of practical research in generating combination tones (from sum and difference frequencies of combined tones) on instruments and demonstrated their existence independently from the hearing process [34]. Modern textbooks under the impression of new experimental results emphasize that combination tones occur inside the ear exclusively, often emphasizing theories of purely "subjective combination tones". Recent experiments by LOHRI at the University of Music and Performing Arts Vienna nevertheless strongly 
suggest the objective reality of combination tones and lead to the conclusion that materials inside musical instruments with freely vibrating components could be acting as non-linear media [34]. It has been shown that linear beating also is of importance in non-linear heterodyne ${ }^{11}$ detection schemes and that at least in heterodyning "until now it has not been realized that the standard textbook equation for mixing usually fails, as in reality, almost all mixers contain a beating stage at their input [35]". It is important to keep in mind that the above simplifying discussion of sum and difference frequencies only touched on one aspect of mixing products of non-linear media when frequencies are combined. Other products comprise integer multiples of the input frequencies, called harmonics and sum and differences between different harmonics, as well as mixtures between harmonics, harmonic combination frequencies and combination frequencies of the fundamental frequencies and harmonics. Higher-order mixing products are called intermodulation frequencies, and their generation cannot be visualized but only calculated using convolution. In non-linearly mixed frequencies, the components from which the carrying frequency emerged lose signal character. In a Fourier transform of the temporal development of the curve (time domain), only the carrier and the modulating frequency can be resolved (in frequency domain). Sum and difference frequencies together with higher-order harmonics of the fundamental frequency and intermodulation products are the products of non-linear frequency mixing in more complex mixing processes, such as in mixers for radio frequencies. In a way, it can be said that non-linear mixing between periodic frequencies is among if not the most basic process in physical evolution in which a dynamic whole can be generated where "the whole is more than the sum of its parts" but with the concession that the original "parts" cannot be easily recovered from the addition as soon as it developed into a non-linear mixing. Probability has been transmitted from the input parts to the output. Non-linearity that originates in the medium based on "own" structural dispositions for the behavior can cause differential amplification and redistribution between amplitudes. Some examples of non-linear behaviors (wave guiding and frequency mixing) and how non-linearity is manifested in mathematical form (in the simplest case of beating and generation of sum and difference frequency components of the fundamental without further higher-order sidebands and harmonics of the fundamental) have been discussed here. Some interesting questions from an evolutionary perspective are, of course, how can mechanisms that "handle" waves of different amplitudes or frequencies in different ways be envisioned? How can mathematical non-linearities be physically effective in structures? What could a structural disposition for the capacity of non-linear frequency mixing possibly look like? ${ }^{12}$ According to the research done for the paper, these questions cannot yet be answered by science or at least have not driven interest in a more detailed answer.

\subsection{The Importance of Non-Linearities}

It is known from spectroscopy that types of motion of (charged) particles on an atomic scale are equal to quantized (discrete) energy levels. Minute changes, which are measurable as changes in energy, translate into changes in the probability of motion as translation, rotation, vibration or electronic energy level transitions or even of a transformation from one type into another. The connection between the form of the medium and the motions excitable therein, especially of repeated correlated motions around positions of rest is strong. In an isotropic medium, it is assumed that all quadratic contributions to total energy, known under the name kinetic energy $\left(\mathrm{E}_{\mathrm{kin}}\right) \mathrm{mv}^{2}$, or $\mathrm{qr}^{2}$, amount to the same average value, given as proportional to $\mathrm{k}_{\mathrm{B}} \mathrm{T}$ (equipartition theorem; $\mathrm{K}_{\mathrm{B}}$ being Boltzmann's constant) [36]. Total energy is then equally distributed over all (excitable) energy levels and associated degrees of freedom (dofs) of motion. This makes temperature $\mathrm{T}$ of a medium proportional to the mean kinetic energy of its particle motions in thermodynamic equilibrium. Inside an ensemble of a closed system (i.e., which is not coupled to a surrounding system with a different average value (different $\mathrm{T}$ )), many different isoenergetic configurations of the ensemble of particle motions, so-called microstates, lead to the same temperature value. Although microstates of ensembles of chemical molecules have more degrees of 
freedom (dof) than a cyclically periodic sinusoid wave and the phase space thereby becomes accordingly more complex, the analogy between the incoherent relative phase in a spectrum of waves leading to an average local intensity on a stricken screen and many different configurations inside a closed ensemble of particle motion leading to a common average $\mathrm{E}_{\mathrm{kin}}$ is astonishing. The influence of the relative phase altering the distribution of intensity in space on frequencies thereby not altering the (theoretical) average energy per length of a frequency's period transmissible to an area of impact but completely changing local availability of energy is pictorial for imagination. Transmitted total energy is the same, but locally there are differences from the average which forms a global intensity distribution pattern (interference pattern). The statistical probability measure of thermodynamics distinguishes microstates (of independent motions in physical phase space) from each other in energetic terms. The distinction of energetically equivalent different relative phases, in contrast, is possible by the image of their intensity distributions on a stricken surface, i.e., the spatio-temporal pattern given to the energy of the field. When non-linear processes come into play-as in the described emergence of solitons and mixing processes between superposed frequencies - equipartition is counteracted in a locally and temporally limited way. The spectrum and most certainly also the average value of the ensemble is altered. Thereby-limited in time and space-anisotropic distributions are compatible with and thus functional for maintenance of the structure of the medium. It is trivial to mention that the other accounts on function would not distinguish the physical process from transmission of a random pattern and thus treat anisotropic media not differently from isotropic media regarding their properties to partly store and re-emit received wavefield information.

Thanks to the basic function of maintaining medium structure under reversible excitation of non-linearly emerged new frequencies (mixing) or under reversible excitation of a solitonic balance between dispersion and non-linear differential acceleration or enhancement ${ }^{13}$ of vibrational modes, information in its basic form can be embodied and stored.

\subsection{Information in Its Most Basic Physical Representation}

Information in its most basic form must be processed-in a basic way-in physical processes, i.e., processes under energy transformations and changes in position. Information is not matter and not energy [37]. In fact, it is proposed here in reference to STONIER $[18,38,39]$ and taking into account what has been said about basic (maintenance) functionality to consider information in connection with energy transformation processes. As has been shown in the discussion about a basic form of physical self-referentiality in the coupling of non-linear processes to linear interference, an image of spatio-temporal distribution of energy per period on a stricken surface can be transmitted via fields, or rather, media, by wave dynamics. Connecting to the questions asked in the introduction, we can propose a tentative answer for the physical selective regime of evolution: a difference in physical structures that-from the view of the maintenance account-can make a difference is this basic form of physical self-referentiality. The meaning of an image transmitted via a medium leading to a non-linear dissipative organization of wave-dynamically relevant properties (such as RI) according to the image's patterning for energy made available as energy per period and area $\left[\mathrm{J} / \mathrm{sm}^{2}\right]$ can be given by the maintenance account. The function of the otherwise immaterial image is a contribution to the maintenance or repeated generation of distribution functions similar to "itself". One special property which wave transmission of pacing for local energy availability has is its potential independence from the type of medium it is transmitted by. Naturally in transmission from one medium into another, details of the information of the energy pacing are lost, but the natural relation between electromagnetic waves in the electromagnetic field and moving as well as resting charges (as described by Maxwell's equations) facilitates cross-medium transmission. Information should be-at least in principle—quantifiable as the amount of discrete possibilities to distribute probability for selection. 
When many options to choose from are available, e.g., when in the case of binary coin throws many coins are thrown, information necessary to describe the sequence of decision events will be a lot. Although the result might resemble a random sequence of decision events, information that can be used in communication as quantified by SHANNON's formula (for information entropy, $\mathrm{H}$ on p.14 of [40]) increases when there exist many mutually independent decision events in a sequence. If there are redundancies such as when many decision events are the same and their relative position in the sequence is not important or can be expressed by a short coded additional information, the amount can decrease. In the extreme case of 10 decision events in favor of heads out of ten throws, the probability for the unbiased result is as low as for any other sequence, but the information could be expressed in algorithmic form quite easily as $10 \times$ binary decision event heads provided that the code is available.

A difference that physically makes a difference is primarily something that can accelerate masses or charges; however, this alone is not information but force. A beam of wood that has been mounted so that it can swing when driven under force will oscillate around its resting position back and forth until the energy (force times amplitude of motion away from resting position) is fully transformed into heat and micro motions. In contrast, a pendulum of colliding balls (called Newton's Cradle) can store the number of initially moved balls until shortly before thermalization. With this system, one could transmit-previously mutually coded-information. That is, a pattern of energy availability in space and time can be transmitted by mutually independent units consolidated via a limiting structure when the coupling between them is altered by applying force differentially.

What about the other form of energy, the potential energy, $\mathrm{E}_{\text {pot }}$ ? It behaves complementary to kinetic energy in that it comprises the position of mass and charges in relation to accelerating influences that potentially can be transformed into kinetic energy during relaxation into their resting position. $E_{\text {kin }}$ and $E_{\text {pot }}$ are sufficiently known from physics, but is it possible to logically relate them to the amount of possibilities to choose from for differential acceleration, or, the position of differentially accelerating influences? The soliton wave can conduct energy and the length of its period: if a series of solitons is excited, power and frequency are transmissible in a stable form. The condition for a signal, a wave function that can itself be a discrete sinusoid or comprise discrete elements stable in form, is given. Form stability in waves would have to be translated as stability in energy per frequency. As is widely known, Planck's formula for quanta of black body radiation $(E=h f)$ gives the smallest unit of discrete waves of stable energy/frequency, the constant $h$. Interestingly, the physical quantity $E / f$, that is, $E^{*} \tau$ (with $\tau$ being the duration for a process from a starting point to an endpoint), has meaning for physics. It is called action (therefore Planck's constant $\mathrm{h}$ is called the quantum of action) and is the main quantity in a natural principle, called the principle of least (in strictly non-dissipative systems replaced by "stationary") action (PLA). Processes without dissipation according to the PLA happen along trajectories that are the shortest path (in traveling light this can be taken literally as shortest in time) that connects starting point and endpoint, so-called geodesics.

When in linear classical mechanical systems the relation between $\mathrm{E}_{\mathrm{kin}}$ and $\mathrm{E}_{\text {pot }}$, quantified in a single number as the Lagrangian temporal integral functional over $\left(\mathrm{E}_{\mathrm{kin}}-\mathrm{E}_{\mathrm{pot}}\right)$ [39], changes, physical action changes, and the trajectory deviates from the geodesic. The thrilling fact that this quantity, physical action can be expressed using the units of classical translation (distance and time) as well as of classical wave motion (wave number and frequency) is a detail that has been keeping physics busy for quite some time [41-43] and that most certainly will be of great relevance for insights regarding physical information processing in evolution. An important question in this context is the importance of the connection between linear and non-linear dynamics for evolution. If the group of ANNILA should be right with the theory of a connection between the Second Law of Thermodynamics and the PLA, delineating dispersal of energy along the steepest directional descents in evolution [44], implications for the understanding of natural processes can hardly be imagined. 
According to our considerations of kinetic energy, information changes when a motion structure is reversibly excited; information changes if the input energy makes a difference that manifests as a temporarily stable (almost, given quantum uncertainty) number of correlated medium elements, which potentially can be read out (is potentially communicable) by modulation of an incoming carrier wave. Such a difference can influence further selective processes and lead to further non-linear differential accelerations and enhancements in sensible structures of its surroundings. Information in its basic form can therefore be understood as a BATESONian difference which can be stored (as a relative phase in a field or as in a configuration of chemically bound particles) and which can influence selective processes (differential acceleration, enhancement, delocalization or more generally differential support or weakening of fluctuations present in the receiving system).Translated into action of information on the mechanically relevant kinetic and potential energies, there is:

Kinetic information, which is connected to information contained in kinetic energy. It is the differential enhancement of coherence between mutually independent particle motions in phase space, that is, waves in a relative phase in a wave forming a motion structure with a (communicable) spatio-temporal pattern of interference "coded" intensity related values. In a (projectile) motion of single-particle objects, where $\mathrm{N}_{\text {ensemble }}=1$, speed and direction of propagation are also given by kinetic information, $\mathrm{I}_{\text {kin }}$. If $\mathrm{E}_{\mathrm{kin}}$ occurs without $\mathrm{I}_{\mathrm{kin}}$, it plainly transmits an averaged intensity value anywhere into a stricken target, i.e., it is thermalized.

Thereby kinetic information is related to its complementary in a way analogous to the relationship between kinetic and potential energy:

Structural information ${ }^{14}$ is connected to information contained in potential energy. It amounts to relative position inside a potential as well as to the basic units of potential, charge and mass. Relative position in space and in relation to accelerating (positively or negatively) influences organizes potential energy of correlated densities of mass and correlated densities of charge into spatial networks of distinct geometry. Thresholds for the spatial constraints are defined by topologically influenced restoring forces and local potentials. Subthreshold impacts of energy are reversible due to restoring forces. $E_{\text {pot }}$ without $\mathrm{I}_{\text {Struc }}$ is hardly possible. Even the densest packing of mass or charge defines a spatial structure and threshold energies. Collapse into a point is forbidden by the Pauli principle. Maximum random dispersion into free space under thermalized $\mathrm{E}_{\mathrm{kin}}$, splitting all relations that define elementary structure would minimize $\mathrm{I}_{\text {struc }}$ but also $\mathrm{E}_{\mathrm{pot}}$.

High content of structural information in elements of media can severely alter the kinetic information that a wave entering from a medium with maximum phase velocity initially had. Due to the networks between charges and masses, dense partitions of the space into local cavities occur. Cavities of different scales influence properties of (re)excitable waves by changing fundamentals and associated harmonic spectra of linearly excitable waves. Non-linear effects can also lead to occurrence of new frequencies, and the supply of resonance enhancements for various frequencies from the differently scaled cavities facilitates chaotic developments.

Similarly, motion structures inside a supportive medium can irreversibly alter the structural information of the medium that is carrying them. Optical fibers can be shown to become irreversibly altered in their structure and potentials by propagating solitons in a way that similarly structured solitons out of later incoming light beams will be forming with high probability [45-47].

Kinetic information can only interact with kinetic information via structural information. Frequency mixing can only occur from linear superposition of frequencies via non-linear effects of the medium. Structural information can communicate and act on other structural information via kinetic information and kinetic energy, which usually ought to comprise organizing (kinetic) information such as propagation direction and correlation between motions when supposed to be doing work. Structural information imagined in a world with no kinetic information but only subjected to non-directional, 
non-patterned random thermal motion is most likely to remain incapable of adapting its structure. Kinetic information without structural information to non-linearly support its inherently fluctuating numbers of quanta moving in a correlated phase would thermalize.

Structural information, $\mathrm{I}_{\text {Struc, }}$ cannot inform a receiver unless the receiver is coupled to it via a field or motion containing $\mathrm{I}_{\mathrm{kin}}$. Sensory functions that can receive information about structure from signals in the field in a structure can improve the transmission.

Motions that do not keep the physical action stationary are connected to prolonged paths, additional events of acceleration and dissipation of energy from and into the surrounding environment, i.e., non-linearity or physical impossibility. They might be made possible for a system but bear risks for the maintenance of structure and structural information when externally input kinetic energy cannot be dissipated into the environment and-following the Second Law of Thermodynamics—is dispersing between all available internal dofs that the structural information is supporting and additionally over further dofs made available by partial or complete disintegration of the structure. Thus they make the system dependent on compensating external, i.e., environmental influences for its motion and possibly for maintenance.

Compared to function, information is not self-referential by itself. While structural information can be maintained comparatively invariant to disturbances from environmental kinetic energies ${ }^{15}$, kinetic information is easily destabilized by structures in its environment. Kinetic information too can have a maintenance function, having an influence on being kept largely invariant and potentially re-excited inside its environment. However, different from its structural complementary, it is in need of a balance between the dispersion of motions in a medium (dispersive relation) and the medium's non-linearity. Examples where this is realized are solitons and localized structures [45] which are viable exclusively inside supportive media.

\subsection{Selection Regimes Guiding and Defining Functionality}

In the canal example, the system has the property of coupling the non-linear steepening behavior to the velocity-dependent dispersive behavior for waves of different amplitudes.

The solitary wave is not having a function in the sense of either the etiological or the systemic account. Given the structure of the canal-ship system, the generation of a soliton wave as a means to transport energy in response to a certain kind of excitation and thereby being maintained in its form is simply its relational function, $f(x)$. The system can be excited into this state and return to its initial state-neither ships nor the canal components are structurally changed by the soliton generation process. Generation of solitons is a characteristic, repeatable reaction to a certain type of excitation. Function theory would call this an accidental function, but from an evolutionary perspective, calling it that way is a tool-use-thinking-based hindrance for intuitive thinking ${ }^{16}$ about possible mechanisms for prebiotic evolution. The maintenance account cannot do more than acknowledging stability in $\mathrm{I}_{\text {struc }}{ }^{17}$ and a very slight form of self-referentiality between frequencies excited and spatial frequency of cavities in the canal structure that define the fundamental and its harmonic series. Nevertheless, this slight form of self-referentiality in differential enhancement of amplitude for frequencies inside a non-linear process is based on a principle that allows much stronger basic physical self-referentiality in evolution: the linear generation of a pattern or average value in local availability of energy per period and area coupled to a non-linear differential enhancement of amplitude of frequencies in a spectrum.

Consider the observation by Russell as a structurally disposed property of the evolutionary regime of selection based on human intentional decision making (the selection regime of intentional decision making (idm)), a selection regime which evolved lateviewed along the course of evolution from the early days of planet Earth. In this selection regime, some people, such as the individual John Scott Russell, had the individual and cultural background in 1834 for focusing their attention on the solitary wave observation and to realize its special characteristic (maybe immediately having an intuition for affordances). Civil engineer John Scott Russell out of a set of individuals capable of extracting 
the structurally based function of the canal for future cultural function in the human idm selection regime on this day, together with a portion of a happy accident, enabled the evolution of the function of solitons inside the idm selection regime. The contribution of randomness was, however, strongly reduced already by the ship-canal system structure having the property of generating solitons as an inherent maintenance function and by the cultural human environment that enabled growing up as a naval engineer and having the genetic, phenotypic and experience background for understanding the importance and potential for future affordances of the observed phenomenon.

Thanks to the complex properties of living (human) systems, the soliton motion structure became more than $\mathrm{f}(\mathrm{x})$ of the canal or a weak form of self-referentiality. It could be described as an accidental function in accordance with existing accounts of function (etiological and systemic), but hopefully, it was possible to show that description of its function according to the physical-maintenance-based account is adding to the understanding of the function by enabling an evolutionary perspective. The structure became functional as a tool in idm, causing its maintenance and repeated reproduction in a variety of different media. In the idm selection regime, a difference that arises due to a structurally based inherent (physical maintenance or relational) function can be recognized and selected by the intention-based action of individuals in cultural context and societies, causing functionality according to the maintenance account on a higher level. According to systemic and etiological accounts of function, solitons became functional as a tool or expansion of cultural knowledge. According to the maintenance account, solitons evolved in functionality and are in the idm regime reproduced more often and more in diverse contexts compared to the physical selection regime due to techniques only available through idm. Technically generated solitons can be gained using largely different artificially generated structural dispositions. Solitons used in technical communication e.g. are possible in a variety of different non-linear materials [46]. Only the maintenance account can point out this connection.

Becoming a biologically positively selected (fitness-enhancing) part for a physical structure such as a certain spectral composition or spatiotemporal pattern is highly influential on the probability and frequency of its structural disposition to be maintained or reproduced, according to the maintenance account. The maintenance account focuses on the effect of a potential function regarding its own maintenance by a selective environment. To be maintained by the surrounding environment, a spectrum needs to not trigger non-linear processes that would alter the structural disposition that is bearing it. The biological selective regime evolved many different species and as the systemic and selected effects function accounts, the maintenance account succeeds in recognizing functionality of a structure that is positively selected across species, such as photonic structures [48] that generate, e.g., interference-based structural coloring that occur in bacteria [49], insects [50,51], plants [52], birds [53], mammals [54] and human artifacts [55]. Already in this example of a structural disposition that is widely maintained and reproduced in selection regimes of living systems, the ascription of functionality because of the color generation is unclear $[56,57]$. The maintenance account explains the global reoccurrence of structural coloring functionality in biotic and abiotic (e.g., labradorite, opal and technical) structures such as that of unremarkable random patterned structures by the fact that their underlying dispositions do not cause effects that lead to non-linear reorganization under irreversible loss of the effect-bearing disposition-the basis for more complex functionality. What is it that a structure contributes to be stable? Is it not "just existing"? Most of the dispositions leading to chemical transformations seem to be no functions because their cause gets annihilated by their own effect. Most of the chemical dispositions influence the existence of structures in the surroundings more than that of the bearer. From what has been discussed here, we can give four-necessarily incomplete-answers:

In physical selective regimes, images of intensity distribution can be transmitted, influencing the structural information of receiving media. Functional traits for weak self-referentiality support imaging and enhance transfer over different media. 
In physico-chemical selective regimes, molecules can aggregate into chemical milieus with stabilized local selective properties, generating closure and micro-environments. Functional traits particularly act on forming a stabilizing environment.

In biological selective regimes, structural genetic information reciprocally couples to biochemical organization in its unit of life, the cell. The body evolves as a complex structured environment of nested environments, enabling sensory functions for its own state and signals in its environment. Individuality of systems emerges.

In intentional selective regimes, senses, nervous systems and storage capacity for experiences in the brain are coupled. This enables an individuality that not only models the environment by the signals it is receiving but also creates a selective regime that can generate individual goals. Cooperation with other individuals is enabled on an unprecedented scale of using information and experiences of multiple generations of conspecifics and foreign species. Functionality is used, analyzed and designed in artifacts.

Individuality increasingly develops the more experiences and individual habits can be stored and transmitted, and this is independent from egoistic or altruistic behavior of individuals. The effect of received information needs to be storable to make a difference in the receiving system. As has been discussed, kinetic information for itself can be stable for some time in its field, but to become distributable into different environments and in-between environments, it needs structural information. Maintenance is therefore a function of material structural dispositions or of a coupling between $\mathrm{I}_{\text {kin }}$ and $\mathrm{I}_{\text {Struc }}$. In this paper, the focus has been on the maintenance functionality of the latter, since stability of matter is known from everyday experience. The maintenance account can assist in understanding the contribution of structural dispositions to the coupling between $\mathrm{I}_{\text {kin }}$ and $\mathrm{I}_{\text {Struc }}$ in the process of evolution. Storability is the basis for the original background of Bateson's famous quote ${ }^{18}$ where the difference that is made can do so as it leads to a change in the receiver's mindset. With the evolution of robust means of information storage and processing, genetic storage, the human mind and its brain came into existence.

Table 1 shows an overview of selective regimes that can be differentiated based on impossible structurally disposed properties in the previous regime, which mark the transition to the respective subsequent selection regime, when occurring.

Table 1. Selection regimes in the course of evolution of information processing systems on Earth.

\begin{tabular}{|c|c|c|c|c|}
\hline & \multicolumn{4}{|c|}{ Selective Regime/Evolving Frame of Reference } \\
\hline & Physical & Physico-Chemical & $\begin{array}{c}\text { Metabolico-Genetic } \\
\text { Biological }\end{array}$ & $\begin{array}{c}\text { Intentional Decision } \\
\text { Making }\end{array}$ \\
\hline $\begin{array}{l}\text { Drives for transformation } \\
\text { processes inside } \\
\text { selection regime }\end{array}$ & $\begin{array}{l}\text { Second Law of } \\
\text { Thermodynamics and } \\
\text { principle of least action } \\
\text { linear and non-linear } \\
\text { combinations }\end{array}$ & $\begin{array}{l}\text { Delocalization of } \\
\text { charges and energy; } \\
\text { minimization of } \\
\text { Gibbs free energy }\end{array}$ & $\begin{array}{l}\text { Darwinian natural } \\
\text { selection: fitness and } \\
\text { evolvability; } \\
\text { individual's felt } \\
\text { deviation from } \\
\text { homeostasis at possible } \\
\text { relative minimum }\end{array}$ & $\begin{array}{l}\text { Leading a good life that } \\
\text { makes sense; making } \\
\text { things easy but keeping } \\
\text { them interesting }\end{array}$ \\
\hline $\begin{array}{l}\text { Impossible properties } \\
\text { that mark transition to } \\
\text { subsequent selection } \\
\text { regime }\end{array}$ & $\begin{array}{l}\text { Reproduction; } \\
\text { autocatalysis }\end{array}$ & $\begin{array}{l}\text { Autopoiesis; search } \\
\text { for gradients }\end{array}$ & Teleology & Breaking physical law? \\
\hline
\end{tabular}

Evolution is a multi-level recursive process. Inside complex bodies or even larger evolving structures, selective environments from all of the preceding evolutionary steps are nested.

\section{Discussion}

First of all, the use of the word evolution in the context of physical evolution needs to be explained, considering the fact that the term is used differently in physics and in biology. DARWIN in 1882 in a letter to G. C. WALLICH wrote that "(...) the principle of continuity renders it probable that the principle of life will hereafter be shown to be a part, or consequence of some general law; but this is only conjecture and not science [58]." In Darwin's times, this was a daring conjecture indeed. Given today's scientific experience and knowledge, evolution even in 
physics where it simply refers to changes over time can be said to usually be connected to some kind of invariance or stability in a set plus a selective influence on that set or system from the surroundings. As was elaborated in [18], the large advantage of an evolutionary view is that reductionism is "only" needed for finding the possible base of a structural disposition in a potential landscape or motion structure. As biology learned by the theory of evolutionary development (EvoDevo), derived states or variants of products can often be traced back to (reversible) changes in state of the generating microenvironment. These transitions in state are triggered by altered influences from the surrounding environment. Both products from the microenvironment are its relational functions, but depending on its initial condition, the state, the so-called wild type or the variant, is produced, i.e., is a stable outcome. With an evolutionary perspective, one is looking at evolving units inside selective regimes whose criteria of selection may themselves be subject to evolution. Different selection regimes can be treated modularly and in nested, recursive form. The coupling between units and environment is important as is the creation of new selective criteria. Structurally disposed properties become functional or not; all functionality is based on the basic one, the maintenance inside the selective environment.

To address the question about what differences the living state made to the evolution of matter and fields, the following arguments can be stated. From what has been summarized at the end of Section 2.1, linear interference and averaging processes can generate patterns of intensities and average intensities, and locally high-activation energy thresholds can be surmounted. Thus non-linear physical and physico-chemical processes of dissipative energy exchange can be spatially patterned. The coupling between linear, i.e., stationary processes and non-linear evolving processes can generate physical selection regimes that are locally differing in their "preferences". Repeated transmission of structural information via modulation of carrier wave fields is a poor means of influencing maintenance inside a globally involving environment compared to mechanisms that evolved later, but it is the basis for reproduction.

The living state of matter and fields has achieved a very close coupling between matter and its structural information to excitable kinetic-information-borne motion structures in fields and moving matter, which strongly reduced dependence on chance events for evolution. A recursively effective sequence of environments and different selection regimes evolved as a consequence of different means of storage and processing of structural as well as kinetic information. Different selection criteria for increasing (or decreasing) the probability of structurally disposed states and properties to be maintained or even reproduced inside their environments have led to a diversity of nested environments and generation of complex multi-leveled properties and functions. Linear and non-linear mixing processes have become associated with exclusively biologically produced and reproduced structural information. Due to the evolution of the selection regime of intention-based decision making, not only physical but also purely rational structures and selective criteria as well as functions have been developed, which are starting to surmount constraints for local accumulation of impact on environments by individuals given by physical laws. Artifacts developed from abiotic and biotic materials by human intentional decision making, together with biological processes, are starting to establish new selection criteria which would not have been possible before life and before humans evolved.

The maintenance account is not dependent on explaining the mechanism for why a property is suddenly recognized as functional by some selective mechanism, and the fact that the probability of the property being selectively maintained or even reproduced in any selective regime in relation to before this selective regime developed is sufficient. The maintenance account is, like others, especially well suited for explaining functionality due to its usability as a tool in living systems. Different from the other accounts, it can give a reason for why the distinctive structural-pattern-based effect occurs on Earth at all and possibly its commonness. In combination with the other function accounts, a more thorough analysis of how functions can evolve is possible. In an evolutionary process, even a function which in the sense of the maintenance account is apparently paradoxical 
is possible. Chemical molecules with structurally disposed high reactivity according to the maintenance account which considers short time spans-short in relation to the mean lifetime of the molecule until it reacts-are not functional regarding their structure. An "individual" molecule loses part of its structurally disposed chemical properties when reacting to become a different chemical structure. On a larger timescale, their structurally disposed property, high reactivity can, nevertheless, become functional-from a maintenance account to function definition rising to more complex forms of function according to other accounts of functionality. Inside systems of interacting chemical particles with constrained thermodynamic openness, the structural information that grounds the high reactivity can on a "higher" level of selection influence the chemical milieu of reaction products, shifting the probability for chemical reactions to favoring the reactive molecule, thus secondarily becoming functional regarding structure; e.g., among chemical elements, many isotopes of element technetium cause themselves to have comparatively very short half-lives of less than an hour and are radioactive. In any environments before the human cultural environment, this is a non-functional property in relation to ${ }^{43} \mathrm{Tc}$, causing rareness of those isotopes. The human cultural selective regime found a use for, e.g., the radioactivity and its effects; therefore certain isotopes (e.g., ${ }^{97} \mathrm{Tc}$ ) including synthetically generated radioisotopes (e.g., ${ }^{95} \mathrm{Tc}$ ) of the element are produced. The maintenance account thus can support the tool-use-function argument, since the probability for being reproduced (maintenance in this example cannot be influenced selectively) increased in comparison to pre-human selective regimes and radioisotopes which in environments of planet Earth before human culture and technical capabilities developed to that point could not emerge now occur due to regular artificial production. By adding non-negative self-referentiality to the relational functionality, the maintenance account can examine the development of effects of structural dispositions which initially proved detrimental for their maintenance in the course of evolution. Atoms and molecules show non-negative self-referentiality in many variants of structural dispositions. The maintenance account can explain their primary maintenance function as well as a secondary evolving maintenance of structurally disposed properties or structural dispositions when the selective criteria in the environment are changing. Mechanisms of compensation for negative effects by environmental influences can change non-functional properties into functional ones or enable effects that are only coded together with detrimental effects in a structural disposition to outweigh the disadvantage. Detrimental effects of maintained structures are certainly an important selective factor; maintained means functional due to maintenance account. In living systems, it is important to consider the difference between functionality for maintenance of a disposition or for maintenance of the bearer of that disposition. The best way of detecting this difference is asking whether a detrimental disposition that (potentially) is life-threatening for its bearer could become functional and how. Three possible situations are considered.

The detrimental effect takes a long time to appear, compared to the lifetime of the organism carrying the trait (hiding by long-term expression of effect):

1. The recognition of such an effect of a function might occur too late to enable negative selection by the Darwinian natural selection mechanism. One example would be a propensity for using up resources. Another example would be a propensity for (accidentally) rendering impossible the survival of an important compensator of negative effects in a system. A propensity very common in living systems is to locally accumulate metabolical waste products that show threshold toxicity for the species. In an ecosystem with functional biological detritus utilization cycles and enough space, this property is not harmful, with toxicity below the threshold. Nevertheless, in case the recycling system slowly and unnoticeably weakens, the carrying capacity of an ecosystem for waste accumulators can reduce in favor of propensities to nonrandomly dispose of waste. All three propensities can appear as neutral or economical to the individual as long as the resources, or, the producers of resources and space to evade problematic effects, are abundant. 
2. The environmental conditions prevent the negative action of the function by compensation for its effect (hiding by compensation). The biggest difference to functionality in 1 is that life-threatening propensities hidden through compensator action can (in principle) be recognized by systems. Compensated detrimental propensities different from temporally hidden ones can be immediately effective every time the compensation temporarily stops.

3. The structural disposition for a functional effect is invariably coupled to a nonfunctional effect (side-effect) so that there is hardly an alternative to accepting/adapting to the coupled detrimental effect. A famous example is sickle cell anemia which is a negative effect when maintained in the system since circulatory disturbances occur frequently. A positive effect of this deformation of blood cells is malaria resistance. In environments with high infection rates of malaria, side-effect functionality of sickle cell anemia is evident. Another example would be a firecracker or explosives in general: being a functional source for local, short-period thermalized energy in idm, selective regime lets its abstract structural information (in form of recipes) be maintained and lets its structures be repeatedly reproduced. Functional is a certain way of dissolving its structure under energy input.

The maintenance account can spot structural dispositions and structurally disposed properties that are functional in a self-referential way, follow the evolution of their maintenance and - in combination with the other function accounts—aid in spotting potentially problematic functions that are not negatively selected naturally. The example of the soliton was chosen to introduce the subject matter, since it is a structurally disposed frequently occurring and characteristic $\mathrm{I}_{\text {kin }}$ motion structure possible in almost any type of medium if there is a structurally disposed non-linear differential amplification property in balance with dispersion. The soliton $\mathrm{I}_{\mathrm{kin}}$ for its special properties is deliberately generated and used in the idm selection regime as a tool. According to the maintenance account a functionality of the $\mathrm{I}_{\text {kin }}$ has evolved there.

\section{Conclusions}

The question of what differences the living state made to the evolution of matter and fields was elaborated and discussed using a refined concept of functionality and of physical information from an evolutionary perspective. It was found that material particulate structures/potential landscapes and kinetic motion structures both can be viewed in a series of recursively subsequent environmental selection regimes and are strongly influenced by potentials and the motion history. With the help of the maintenance account on function, the mutual relation between structurally disposed properties and the capacity to control excitation of periodic components with decreasing dependence on chance events was elaborated. Inside the given limits of an average-length paper, a number of aspects of the explicit function of life in the evolutionary process were elucidated. The question of the meaning of the living state in an even larger context of a possible universal evolutionary process is, of course, impossible to answer, one reason being that the human understanding of different states of matter, fields and the coupling therein is limited to interpretations of observations made from our position inside the universe. Our sensual systems developed in the evolutionary process on planet Earth. These largely epistemic constraints of observation are enforced by our inability to predict functions and properties of mixtures and interactions before we observe them. It is interesting to remark, though, that many of the observed physical interactions and products of certain relations can be described by highly abstract algorithms and mathematical structures, which can be expressed using largely different coded symbols and numbers. In the example of the soliton or higher-order non-linear mixing, it can be shown that even when experience with structural properties is lacking, the phenomenon can be described and-within limits - behavior can be predicted when mathematical principles have been developed accordingly. Most probably such general dynamical "things", such as non-linear mixing of harmonic waves or the soliton phenomenon which on Earth already show diverse modes 
of realization, as a type of solution to a dynamic equation, did occur in the course of evolution several times. In our analysis, we started from a weak physical self-referentiality of a function that emerges in the coupling between linear- and non-linear processes and a mechanism of imaging distribution of available energy in time and space. Another prebiotic function exists in chemicals that supports maintenance of chemical properties by influencing the chemical milieu inside an enclosed environment. Both functions involve forms of differential enhancement/weakening or prebiotic selectivity. If it were the case, as is assumed here, that in the course of evolution not only several different environments occur and are established but also several different selective regimes, formed under the influence of disposed structures and their functionalities, then observation and signal detection capacity would be decisive. Based on what can be observed from evolution on planet Earth in the context of how the evolutionary process of the living state influenced observable evolution of sensors, recognition and motion in feedback to sensory inputs, one "educated guess" can be made: if the observation (measurement process) and the coupling between the evaluation of sensory inputs and measurements to actions was a phenomenon with general importance in universal evolution, then the living state (on Earth), evolving its complex sensory systems, its capacity of symbolic abstraction and the production of artifacts capable of computing, definitely contributed to this phenomenon's diversification, heavily influencing the local evolutionary process; if the above assumption is true, maybe the option of leaving imprints on the direction of the process (so it exists) in systems in reach (of Earth) is too.

Funding: This research received no external funding.

Conflicts of Interest: The author declares no conflict of interest.

\section{Notes}

1 When v. Uexküll spoke about environments or "Umwelten" of animals, he pointed out the individual character and relative invariance of it as perceived by the animal [11]. The environment is more defined by being a source of perceivable conditions than by its location.

2 Selective environments, that is, environmental selection regimes, are discussed in Section 2.5.

3 Functional adaptations for being maintained, not-detected and stably transferred during life cycles by hosts.

4 See footnote 2.

5 Or alternatively, wave diffraction: wave packets propagating in a linear medium have a natural tendency to broaden in time (dispersion) and space (diffraction) [19].

6 Respectively, the speed of the water flow dragged by the ship which continued propagation after the ship suddenly stopped-in the case of Russel's observation.

7 In the simple one-dimensional case of waves on a string, differential excitability of frequencies is easily observed: try exciting even multiples of a fundamental (frequency) in a string that is fixed at one end and free to move on the other-it is forbidden by nature. The decisive factor is the form of the fundamental in the given space (the string), namely how nodes (minimum amplitude) and antinodes (maximum amplitude) are distributed at their boundaries.

8 Another common intensity-related measure of available energy from an impacting field is radiance $L(r, \omega)$. It is defined as the power radiated at a given point $r$ in a given direction $\omega$ per unit of projected area perpendicular to that direction per unit solid angle (in steradian or square radian, sr) for a given frequency [W/ $\left.\mathrm{m}^{2} \mathrm{sr}\right]$ [29].

9 More generally a re-emitter, since the process could be a reflection as discussed here or similarly absorption and re-emission or transmission and transition into a medium with an altered RI. The principle is the same.

10 For mathematical reasons, the sum and difference frequencies are $\left(f_{1}+f_{2}\right) / 2$ and $\left(f_{1}-f_{2}\right) / 2$, respectively. In contrast, the audible intensity beating frequency $\left(f_{\text {beat }}\right)$ is double the difference frequency, i.e., $f_{\text {beat }}=\left|f_{1}-f_{2}\right|$, since, for intensity oscillations, it is irrelevant whether the amplitude is above or below the baseline. For readers who are interested in more details: The mathematical reason for a multiplicative mixing occurring when the non-linearity has an exponential, at least quadratic characteristic at least for a technical mixer which includes diode elements can be easily explained. There the sum of two input signals is applied to a diode, where an output current is generated that is dependent on the input voltage, i.e. it is a relational function $(\mathrm{f}(\mathrm{x}))$ of the input voltage. When the input thus appears as an exponent in the calculation of the output, the exponential can be expanded in a Taylor series and for small inputs, the series can be approximated by the first terms, that is output $\approx$ (input a + input $b)+\frac{1}{2}$ (input a + input $b)^{2}$. As is known from the binomial theorem expanding the square term yields a multiplicative mixing of input a and input $b$ : $(a+b)^{2}=\left(a^{2}+2 a b+b^{2}\right)$. This is the critical point in understanding the contribution of the non-linearity to mixing. 
Heterodyne is the name given to a signal frequency created by non-linear mixing of two other frequencies using a technique called heterodyning, and it is a term used in communication engineering, e.g., in modulation of radio signals.

12 A more detailed answer than that mixers use one or more diodes and rely on their non-linear relation between voltage $(\mathrm{V})$ and current (I) to provide the multiplying element would be helpful. Which property of the semiconductor (diode) accounts for the non-linear relation in the IV characteristic?

13 Both acceleration and enhancement are meant to be understood in a neutral, analytical way, i.e., positive as well as negative.

14 The author keeps the term structural information as proposed by Stonier to honor his work and also because the term potential information is already in use with a different definition. Stonier in [38] proposed equating $\mathrm{I}_{\text {struk }}$ with $\mathrm{E}_{\text {pot}}$, which is not adopted, since his argument of thermodynamic improbability does not hold for potentials and $E_{p o t}$ in general [18].

Thus showing a kind of relational zero function, $\mathrm{f}(\mathrm{x})$ simply being the keeping up of thresholds against irreversible distortions. Intuitive thinking often is recognizing affordances or mechanism components inferred by analogy (see introduction). I.e., relational zero function, i.e., physical maintenance of $\mathrm{I}_{\text {Struc }}$ keeping up thresholds for structural constraints to dissolution.

18 Although Bateson's “(...) make a difference because the neural pathways along which it [elementary unit of information] travels and is continually transformed are themselves provided with energy." Elementary unit of information viewed from an evolutionary perspective represents a unit of information inside a system that is already at a very highly evolved level of energy-fueled capacity for signal excitation and information processing: the human mind.

\section{References}

1. Wouters, A. The Function Debate in Philosophy. Acta Biotheor. 2005, 53, 123-151. [CrossRef]

2. Beckner, M. The Biological Way of Thought; Columbia University Press: New York, NY, USA, 1959.

3. Delancey, C.S. Ontology and Teleofunctions: A Defense and Revision of the Systematic Account of Teleological Explanation. Synthese 2006, 150, 69-98. [CrossRef]

4. Cummins, R. Functional Analysis. J. Philos. 1975, 72, 741-765. [CrossRef]

5. Mossio, M.; Saborido, C.; Moreno, A. An Organizational Account of Biological Functions. Br. J. Philos. Sci. 2009, 60, 813-841. [CrossRef]

6. Nanay, B. A Modal Theory of Function. J. Philos. 2010, 107, 412-431. Available online: https://www.pdcnet.org/jphil/content/ jphil_2010_0107_0008_0412_0431 (accessed on 26 April 2021). [CrossRef]

7. Neander, K. The teleological notion of 'function'. Australas. J. Philos. 1991, 69, 454-468. [CrossRef]

8. McLaughlin, P. What Functions Explain. In Functional Explanation and Self-Reproducing Systems; Cambridge University Press: Cambridge, UK, 2000.

9. Vermaas, P.E.; Houkes, W. Ascribing Functions to Technical Artefacts: A Challenge to Etiological Accounts of Functions. Br. J. Philos. Sci. 2003, 54, 261-289. [CrossRef]

10. Millikan, R.G. In defense of proper functions. Philos. Sci. 1989, 56, 288-302. [CrossRef]

11. von Uexküll, J.; Mildenberger, F.; Herrmann, B. (Eds.) Umwelt und Innenwelt der Tiere. In Klassische Texte der Wissenschaft, Nachdr. der 2. Aufl. 1921; Springer Spektrum: Berlin, Germany, 2014.

12. von Uexküll, J. Streifzüge durch die Umwelten von Tieren und Menschen. In Ein Bilderbuch unsichtbarer Welten. Bedeutungslehre; Rowohlt: Hamburg, Germany, 1956.

13. Mildenberger, F.; Herrmann, B. Uexküll; Springer: Berlin, Germany, 2014.

14. Maier, J.R.A.; Fadel, G.M. Affordance: The Fundamental Concept in Engineering Design. In Volume 4: 13th International Conference on Design Theory and Methodology; American Society of Mechanical Engineers: New York, NY, USA, 2001; pp. 177-186.

15. Brown, D.C.; Blessing, L. The Relationship between Function and Affordance. In Proceedings of the IDETC/CIE 2005: ASME 2005 International Design Engineering Technical Conferences \& Computers and Information in Engineering Conference, Long Beach, CA, USA, 24-28 September 2005; pp. 155-160. [CrossRef]

16. Gibson, J.J. James. The Discovery of the Occluding Edge and Its Implications for Perception. In The Ecological Approach to Visual Perception; First published in 1986; Taylor \& Francis Group: New York, NY, USA; London, UK, 2015.

17. Bateson, G.; Holl, H.G. Ökologie des Geistes. Anthropologische, Psychologische, Biologische und Epistemologische Perspektiven, 1. Aufl.; Suhrkamp-Taschenbuch Wissenschaft 571; Suhrkamp: Frankfurt am Main, Germany, 1985; ISBN 3518281712.

18. Grathoff, A. Exploration of Structural and Kinetic Components of Physical Information. Proceedings 2020, 47, 16. [CrossRef]

19. Mitchell, M.; Segev, M. Self-trapping of incoherent white light. Nature 1997, 387, 880-883. [CrossRef]

20. Bundgaard, J. A Survey of the History and Properties of Solitons. Available online: https://inside.mines.edu/ \{\}tohno/teaching/ PH505_2011/A-Survey-of-The-History-and-Properties-of-Solitons.pdf (accessed on 26 April 2021).

21. Segev, M.; Stegeman, G. Self-Trapping of Optical Beams: Spatial Solitons. Phys. Today 1998, 51, 42-48. [CrossRef]

22. Soomere, T. Nonlinear ship wake waves as a model of rogue waves and a source of danger to the coastal environment: A review. Oceanologia 2006, 48, 185-202.

23. Yao-Tsu Wu, T. Generation of upstream advancing solitons by moving disturbances. J. Fluid Mech. 1987, 184, 75-99.

24. Jenkins, A. Self-oscillation. Phys. Rep. 2013, 525, 167-222. [CrossRef]

25. Ishii, K.; Adachi, S.; Hayashi, H.; Menshov, I. Lagrangian description of energy conversion in the Taconis oscillations. AIP Conf. Proc. 2015. [CrossRef] 
26. Moore, G.D. Physics of Music Lecture Notes. Available online: https://theorie.ikp.physik.tu-darmstadt.de/qcd/moore/ph224 /lecnotes.pdf (accessed on 26 April 2021).

27. Darwin, C.; Neumann, C.W.; Heberer, G. Die Entstehung der Arten Durch Natürliche Zuchtwahl; [Nachdr.]; Reclams UniversalBibliothek 3071; Reclam: Stuttgart, Germany, 2010.

28. Mayr, E. Animal Species and Evolution; Harvard University Press: Cambridge, MA, USA, 1963.

29. Patow, G.; Pueyo, X. A Survey of Inverse Surface Design from Light Transport Behavior Specification. Comput. Graph. Forum 2005, 24, 773-789. [CrossRef]

30. UCD. Physics 9B-Waves, Sound, Optics, Thermodynamics, and Fluids. 2021. Available online: https://phys.libretexts.org/@go/ page/18373 (accessed on 23 June 2021).

31. Bislin, W. Schwebung: Eine praktische Anwendung der Additionstheoreme. License: Public Domain. Available online: http: / / walter.bislins.ch / blog/index.asp?page=Schwebung\%3A+Eine+praktische+Anwendung+der+Additionstheoreme (accessed on 26 April 2021).

32. Run, M. Beat-Frequency-Derived.svg. License: Creative Commons. Available online: https://commons.wikimedia.org/wiki/File: Beat-frequency-derived.svg (accessed on 26 April 2021).

33. Heller, E.J. Why you hear what you hear. In An Experiential Approach to Sound, Music, and Psychoacoustics; Chapter 23 Pitch Perception; Princeton Univ. Press: Princeton, NJ, USA, 2013; pp. 437-447.

34. Lohri, A.; Carral, S.; Chatziioannou, V. Combination tones in violins. In Proceedings of the Second Vienna Talk, Vienna, Austria, 19-21 September 2010.

35. Verbiest, G.J.; Rost, M.J. Beating beats mixing in heterodyne detection schemes. Nat. Commun. 2015, 6, 6444. [CrossRef]

36. Atkins, P.W.; Trapp, C.A.; de Paula, J.; Cady, M.P.; Giunta, C.; Höpfner, A. Physikalische Chemie; VCH: Weinheim, Germany, 1987.

37. Wiener, N. Cybernetics or Control and Communication in the Animal and the Machine, 2nd ed.; MIT Press: Cambridge, MA, USA, 1965.

38. Stonier, T. Information and the internal structure of the universe. In An Exploration into Information Physics; Springer: London, UK, 1994.

39. Grathoff, A. An Evolutionary View on Function-Based Stability. Proceedings 2017, 1, 54. [CrossRef]

40. Shannon, C.E.; Weaver, W. The Mathematical Theory of Communication; Univ. of Illinois Press: Urbana, IL, USA, 1998.

41. Dirac, P.A.M. The quantum theory of the emission and absorption of radiation. Proc. R. Soc. Lond. A 1927, 114, $243-265$.

42. Feynman, R.P. QED. Die Seltsame Theorie des Lichts und der Materie, Ungekürzte Taschenbuchausgabe; Piper 31316; Piper: München, Germany, 1988.

43. Ogborn, J.; Taylor, E.F. Quantum physics explains Newton's laws of motion. Phys. Educ. 2005, 40, 26-34. [CrossRef]

44. Mäkelä, T.; Annila, A. Natural patterns of energy dispersal. Phys. Life Rev. 2010, 7, 477-498. [CrossRef]

45. Barland, S.; Coen, S.; Erkintalo, M.; Giudici, M.; Javaloyes, J.; Murdoch, S. Temporal localized structures in optical resonators. Adv. Phys. X 2017, 2, 496-517. [CrossRef]

46. Denz, C.; Weilnau, C. Räumliche optische Solitonen-Licht steuert Licht. Phys. J. 2003, 2, 33-39.

47. Saleh, M.F.; Conti, C.; Biancalana, F. Anderson localisation and optical-event horizons in rogue-soliton generation. Opt. Express 2017, 25, 5457-5465. [CrossRef] [PubMed]

48. Vukusic, P.; Sambles, J.R. Photonic structures in biology. Nature 2003, 424, 852-855. [CrossRef]

49. Kientz, B.; Luke, S.; Vukusic, P.; Péteri, R.; Beaudry, C.; Renault, T.; Simon, D.; Mignot, T.; Rosenfeld, E. A unique self-organization of bacterial sub-communities creates iridescence in Cellulophaga lytica colony biofilms. Sci. Rep. 2016, 6, 19906. [CrossRef] [PubMed]

50. Vukusic, P.; Sambles, J.R.; Lawrence, C.R.; Wootton, R.J. Quantified interference and diffraction in single Morpho butterfly scales. Proc. R. Soc. Lond. B 1999, 266, 1403-1411. [CrossRef]

51. Seago, A.E.; Brady, P.; Vigneron, J.-P.; Schultz, T.D. Gold bugs and beyond: A review of iridescence and structural colour mechanisms in beetles (Coleoptera). J. R. Soc. Interface 2009, 6 (Suppl. 2), 165-184. [CrossRef]

52. Glover, B.J.; Whitney, H.M. Structural colour and iridescence in plants: The poorly studied relations of pigment colour. Ann. Bot. 2010, 105, 505-511. [CrossRef]

53. Burg, S.L.; Parnell, A.J. Self-assembling structural colour in nature. J. Physics. Condens. Matter Inst. Phys. J. 2018, $30,413001$. [CrossRef]

54. Prum, R.O.; Torres, R.H. Structural colouration of mammalian skin: Convergent evolution of coherently scattering dermal collagen arrays. J. Exp. Biol. 2004, 207, 2157-2172. [CrossRef]

55. Kim, H.; Ge, J.; Kim, J.; Choi, S.; Lee, H.; Lee, H.; Park, W.; Yin, Y.; Kwon, S. Structural colour printing using a magnetically tunable and lithographically fixable photonic crystal. Nat. Photon 2009, 3, 534-540. [CrossRef]

56. Prum, R.O.; Torres, R. Structural colouration of avian skin: Convergent evolution of coherently scattering dermal collagen arrays. J. Exp. Biol. 2003, 206, 2409-2429. [CrossRef]

57. Lopez-Garcia, M.; Masters, N.; O’Brien, H.E.; Lennon, J.; Atkinson, G.; Cryan, M.J.; Oulton, R.; Whitney, H.M. Light-induced dynamic structural color by intracellular 3D photonic crystals in brown algae. Sci. Adv. 2018, 4, eaan8917. [CrossRef] [PubMed]

58. de Beer, G. Some Unpublished Letters of Charles Darwin. Notes Rec. R. Soc. 1959, 14, 12-66. Available online: https://www.jstor. org/stable/3087184 (accessed on 26 April 2021). 